\title{
Thyroid peroxidase forms thionamide-sensitive homodimers: relevance for immunomodulation of thyroid autoimmunity
}

\author{
David O. McDonald • Simon H. S. Pearce
}

Received: 8 June 2009 /Revised: 1 July 2009 / Accepted: 14 July 2009/Published online: 8 August 2009

(C) The Author(s) 2009. This article is published with open access at Springerlink.com

\begin{abstract}
Thyroid peroxidase (TPO) is the key enzyme in thyroid hormone production and a universal autoantigen in Graves' and other autoimmune thyroid diseases. We wished to explore the expression of TPO and whether it was affected by thionamide antithyroid drugs. We studied recombinant TPO, stably expressed by a Chinese hamster ovary cell line (CHO-TPO) and transiently expressed TPOenhanced green fluorescent protein (eGFP) and -FLAG fusion proteins. Immunoblotting of CHO-TPO cell extracts showed high-molecular weight (HMW) TPO isoforms that were resistant to reduction, as well as $110 \mathrm{kDa}$ monomeric TPO. Co-immunoprecipitation and enzyme-linkedimmunosorbent assay (ELISA) binding studies of FLAGand eGFP-tagged TPO demonstrated TPO dimerisation. CHO-TPO cells cultured in methimazole (MMI) for 10 days showed a significant reduction in HMW-TPO isoforms at MMI concentrations of $1 \mu \mathrm{M}$ and above $(p<0.01)$, whereas monomeric TPO expression was unchanged. We observed a similar reduction in HMW-TPO in CHO-TPO cells cultured in propylthiouracil (10 $\mu \mathrm{M}$ and above). Binding of Graves' disease patient sera and TPO-Fabs to enzymatically active TPO that was captured onto solid phase was not abrogated by MMI. The cellular localisation of TPO in CHO-TPO cells was unchanged by MMI treatment. Our demonstration of homodimeric TPO and the reduction in HMW-TPO isoforms during thionamide treatment of CHO-TPO cells shows, for the first time, an effect of thionamides on TPO structure. This suggests a structural correlate to the effect of thionamides on TPO enzymatic activity and opens up a
\end{abstract}

D. O. McDonald $\cdot$ S. H. S. Pearce $(\bowtie)$

Institute of Human Genetics, Newcastle University,

International Centre for Life,

Central Parkway,

Newcastle upon Tyne NE1 3BZ, UK

e-mail: s.h.s.pearce@ncl.ac.uk novel potential mechanism for thionamide immunomodulation of autoimmune thyroid disease.

Keywords Thyroid peroxidase - Homodimerisation . Methimazole · Propylthiouracil · Graves' disease · Immunomodulation

\section{Introduction}

Thyroid peroxidase (TPO) is the commonest autoantigen for mankind, $5 \%$ to $18 \%$ of individuals in Westernised populations showing circulating anti-TPO antibodies, with an age-related increase in prevalence. Furthermore, antiTPO antibodies are practically universal in individuals with all clinical forms of autoimmune thyroid disease (AITD) [1]. TPO functions as a cell-surface enzyme and is predicted to have four modular domains [2]: an aminoterminal myeloperoxidase (MPO)-like domain, a complement-control protein (CCP)-like domain (also known as a sushi domain), an epidermal growth factor-like domain, and a short carboxy-terminal membrane-anchoring domain. The anti-TPO immune response in AITDs is dominated by antibodies that recognise specific conformational epitopes, termed immunodominant regions, which appear to be composed of structural residues from both its MPO-like and CCP-like domains [3-6]. The enzymatic activity of TPO is essential for thyroid hormone biosynthesis, and molecular abnormalities causing defective TPO activity lead to profound congenital hypothyroidism and goitre [7].

Thionamide compounds, including propylthiouracil (PTU), methimazole (MMI), and carbimazole, have been used to treat Graves' disease and other forms of hyperthyroidism for more than 60 years [8]. Their antithyroid action 
is mediated by their ability to act as a preferential substrate for TPO. The first step in thyroid hormone synthesis is iodination of thyroglobulin tyrosyl residues. In the presence of thionamide, TPO iodinates the sulphenyl group of the thionamide drug, instead of the thyroglobulin tyrosyl residue. During treatment of hyperthyroidism, thionamides accumulate in the thyroid follicular cells over time. Their increasing concentration results in reduced thyroid hormone synthesis and eventually leads to a complete blockade of thyroid hormone production [9]. An additional effect of thionamides is that they may result in a lasting remission from hyperthyroid Graves' disease, during which, certain individuals may become almost indefinitely euthyroid, even after thionamide administration is stopped $[10,11]$. This durable remission of Graves' disease occurs in $40 \%$ to $50 \%$ of patients who are treated with thionamides and appears to occur irrespective of which compound has been administered, or the dose used [12]. Optimal remission rates of hyperthyroid Graves' disease occur after 6 to 18 months of thionamide administration and are associated with a reduction in circulating antithyroid antibodies, including those directed against the thyrotropin receptor (TSHR) and TPO epitopes [13, 14]. This latter, 'remission-inducing' property of thionamides in Graves' hyperthyroidism has been referred to as an immunomodulatory action. While the enzymatic mechanism for the antithyroid action of thionamides is well documented, literature concerning the effects of thionamides on TPO expression or structure is sparse. Similarly, the molecular basis for the widely exploited immunomodulatory action of thionamides in Graves' disease remains unknown, although many hypotheses have been explored $[11,15,16]$. Bearing in mind that TPO is a universal autoantigen in AITD and that the immune responses are directed against conformational TPO epitopes, we set out to explore the hypothesis that thionamides may influence the structure of TPO (i.e., the conformation of these epitopes) or its subcellular localisation.

\section{Materials and methods}

Cell culture, lysis, and protein extraction

A Chinese hamster ovary cell line that had been stably transfected with full-length human TPO (CHO-TPO) was used to generate enzymatically active TPO (a gift from Dr. Monika Gora (Warsaw) and Prof. Paul Banga (London)). CHO-TPO cell monolayers were cultured for 7-10 days in complete media (Hams F12 $+500 \mu \mathrm{g} / \mathrm{ml}$ hygromycin B, $10 \%$ foetal bovine serum). Cells were washed three times in cold phosphate-buffered saline (PBS) before scraping and pelleting. Cell pellets were resuspended in ice cold lysis buffer $(50 \mathrm{mM}$ Tris-HCl, pH7.3; $150 \mathrm{mM} \mathrm{NaCl}$; $5 \mathrm{mM}$ ethylenediaminetetraacetic acid; $10 \%$ glycerol $(w / v)$; $1 \%$ Triton X-100 $(w / v)$, protease inhibitors). After two freeze-thaw cycles, debris was pelleted at $13,000 \mathrm{~g}$ for $30 \mathrm{~min}$ at $4^{\circ} \mathrm{C}$. Supernatants were collected as a source of TPO and stored at $-20^{\circ} \mathrm{C}$. Aliquots of each preparation were tested for peroxidase activity by oxidation of $0.5 \%$ guaiacol in PBS [17].

\section{Immunoblotting}

Recombinant human TPO (rhTPO) was prepared as above with the following exceptions: CHO-TPO cells were cultured either for 10 days in the presence of MMI $(1,10$, 100 , and $500 \mu \mathrm{M})$ in complete media or for 7 days in complete media followed by $50 \mathrm{~h}$ with 6-PTU (Sigma) (10, 100 , and $500 \mu \mathrm{M})$ in serum-free media (OptiMEM). Total protein was standardised by micro-bicinchoninic acid assay (BCA) assay (Pierce labs) and electrophoresed at $20 \mathrm{~mA} /$ gel on sodium dodecyl sulphate (SDS)-polyacrylamide gels after mild denaturation by concomitant incubation with $40 \mathrm{mM}$ iodoacetamide and $50 \mathrm{mM}$ dithiothreitol (DTT) in SDS-loading buffer $(0.1 \mathrm{M}$ TrisCl, pH6.8; $20 \%$ glycerol, $4 \%$ SDS, $0.001 \%$ bromophenol blue) for $10 \mathrm{~min}$ at $97^{\circ} \mathrm{C}$. Alternative denaturation/reduction buffers including DTT only, 2-mercaptoethanol, and iodoacetamide alone were used, as detailed in Fig. 1. Gels were blotted by electrotransfer onto nitrocellulose (Hybond ECL, GE Healthcare) for $90 \mathrm{~min}$ at $90 \mathrm{~V}$, on ice. After transfer, non-specific

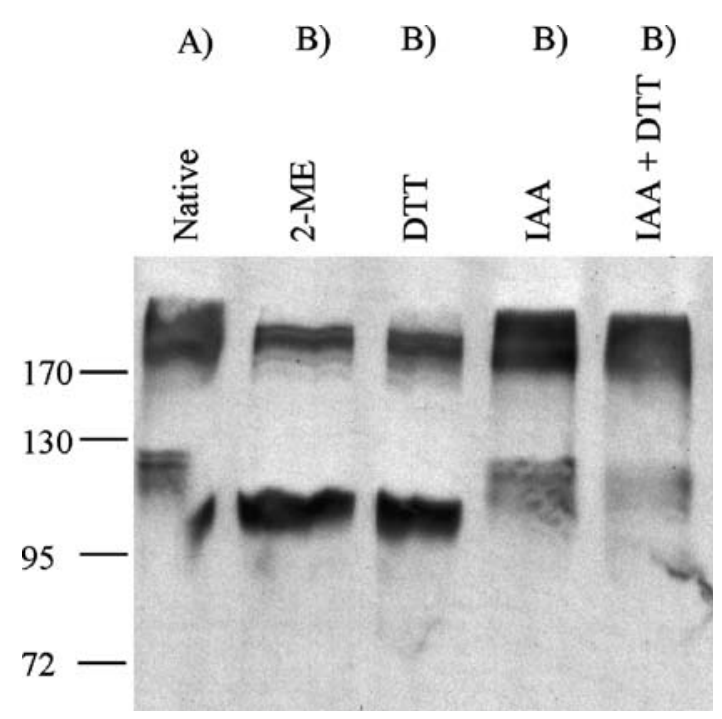

Fig. 1 Recombinant human TPO forms high molecular weight complexes. Cell extracts, native (A) or denatured $\left(\mathrm{B} ; 98^{\circ} \mathrm{C}\right.$ for $10 \mathrm{~min}$ ) with either 2\% 2-ME, $50 \mathrm{mM}$ DTT, $40 \mathrm{mM}$ iodoacetamide (IAA), or $50 \mathrm{mM}$ DTT and $40 \mathrm{mM}$ IAA, from CHO cells stablyexpressing rhTPO were assessed by immunoblotting with mAb47. Bands migrating at $\sim 105-110 \mathrm{kDa}$ were observed in all conditions corresponding to monomeric TPO. High molecular weight TPO isoforms were also detected above $200 \mathrm{kDa}$ 
binding to the membrane was blocked using 5\% non-fat milk in Tris-buffered saline and $0.1 \%$ Tween 20 (TBST) overnight, followed by $16 \mathrm{~h}$ incubation with a monoclonal anti-TPO antibody (mAb47, Alexis Biochemicals, UK) in $1 \%$ bovine serum albumin (BSA) in phosphate-buffered saline and $0.1 \%$ Tween 20 (PBST). Membranes were washed in TBST and incubated for $1 \mathrm{~h}$ with anti-mouse IgG alkaline phosphatase conjugate antibody, raised in goat $(1 / 1,000$ in $1 \%$ BSA in TBST, Pierce) to detect antibody binding. Four 5-min washes followed before adding chemiluminescent substrate (West Dura, Pierce) and exposing to film. Band densitometry was performed using ImageQuant software and normalised for glyceraldehyde 3-phosphate dehydrogenase (GAPDH) expression in each lane.

\section{Cloning eGFP/FLAG-TPO}

Oligonucleotide primers were designed to flank and overlap the $3^{\prime}$ and $5^{\prime}$ ends of TPO cDNA, previously cloned into pcDNA3.1 (gift of Dr. B Rapoport, LA [18]), and introduced a $\mathrm{G}$ to $\mathrm{C}$ base change into the stop codon to allow read-through to C-terminally tagged fusion protein, as well as $\mathrm{XbaI}$ and EcoRI sites for subsequent in-frame cloning into pCMV14-3xFLAG vector (Sigma) or AgeI and EcoRI for pEGFP-N1 (Clontech). The full-length cDNA was polymerase chain reaction (PCR)-amplified using highfidelity Phusion polymerase (NEB, Beverly, MA, USA). The resulting $3 \mathrm{~kb}$ bands were sub-cloned into either the TOPO-pCR-XL (Invitrogen) or pGEM T-easy vectors (Promega) and ligated into their final vectors. Single clones positive for full-length TPO were identified by restriction digest and in-frame integration of the cDNA confirmed by DNA sequencing. FLAG-tagged bacterial alkaline phosphatase (FLAG-BAP) and unconjugated enhanced green fluorescent protein (eGFP; pEGFP-N1) constructs were obtained from manufacturers. The unconjugated eGFP construct will be referred to as eGFP-nil.

\section{Co-immunoprecipitation}

$\mathrm{CHO}$ cells at $70 \%$ confluence were co-transfected with FLAG-TPO, eGFP-TPO $\left(10 \mu \mathrm{g}\right.$ per $10 \mathrm{~cm}^{2}$ plate $)$, or their respective controls: FLAG-BAP, eGFP-nil using Fugene HD (Roche, UK). After $18 \mathrm{~h}$, proteins were extracted as previously described. Input samples were retained, and the remainder pre-cleared for non-specific binding with protein-G sepharose beads for $1 \mathrm{~h}$. Samples were then centrifuged at $1,000 \mathrm{~g}$ at $4^{\circ} \mathrm{C}$ for $1 \mathrm{~min}$, the N-terminally tagged TPO fusion proteins were immunoprecipitated overnight using their corresponding antibodies: AntiFLAG M2 (Sigma), $0.5 \mu \mathrm{g}$, or GFP(FL) and GFP(B2; Santa Cruz Antibodies, CA, USA). Immunoprecipitating beads were thoroughly washed with lysis buffer and subjected to SDS-polyacrylamide gel electrophoresis (PAGE; as above) alongside their corresponding preimmunoprecipitated input samples. Immunoblotting was performed as before with the following exceptions: the presence of eGFP-TPO was detected with anti-GFP murine monoclonal or FLAG-TPO using anti-FLAG M2.

\section{Tagged-TPO ELISA}

Extracts from CHO cells co-transfected with FLAG-TPO, and either eGFP-TPO or eGFP-nil were prepared as above. Samples with equal total protein, assessed by micro-BCA, were incubated overnight in enzyme-linked-immunosorbent assay (ELISA) plates pre-coated with anti-FLAG M2 mouse antibody (100 ng/well) and blocked with 2\% BSA in PBST. After thorough washing with PBST, GFP(FL) rabbit antibody was added for $1 \mathrm{~h}$ and detected with anti-rabbit AP-conjugate. Development of para-nitrophenylphosphate (pNPP) was measured $\left(\mathrm{A}_{405}\right)$ after $1 \mathrm{~h}$.

\section{Bacterial expression of Fab fragments}

Four cloned Fabs, TR1.9 and TR1.8; and WR1.7 and SP1.4, which recognised the human TPO B and A immunodominant epitopes, respectively, were obtained (kind gift of Drs. B Rapoport and S McLachlan, LA) [19]. Starter cultures of XL1-blue Escherichia coli containing each of the Fab expression constructs were used to inoculate Superbroth: $30 \mathrm{~g} / 1$ tryptone, $20 \mathrm{~g} / 1$ yeast extract, $10 \mathrm{~g} / 1 \mathrm{3-(N-morpholino)propanesulfonic} \mathrm{acid,} 20 \mathrm{mM}$ $\mathrm{MgCl}_{2}, 1 \%$ glucose grown at $37^{\circ} \mathrm{C}$ with shaking. At $\mathrm{A}_{595}$ approximately $0.4 \mathrm{Fab}$ production was induced with $1 \mathrm{mM}$ isopropyl $\beta$-D-1-thiogalactopyranoside for $16 \mathrm{~h}$ at $28^{\circ} \mathrm{C}$. Cells were pelleted and after four freeze-thaw cycles were centrifuged at $30,000 \mathrm{~g}$ for $30 \mathrm{~min}$ at $4{ }^{\circ} \mathrm{C}$. The supernatant was retained as a source of Fab.

\section{ELISA for TPO antibody binding}

mAb47 was used to capture recombinant TPO from CHOTPO cell extracts. Binding of Fabs and patient sera to captured TPO was studied in different MMI (Sigma Chemicals, Poole, UK) conditions, with MMI added after Fab or serum binding. Sera that were positive for TPO antibodies on a conventional chemiluminescent assay (ADVIA Centaur, Bayer) from 36 patients with AITD were studied including 30 (29 females) with Graves' disease, and six (five females) with autoimmune hypothyroidism (mean age 47 years, range 18-78). All sera were collected with the approval of the Northumberland Tyne and Wear local research ethics committee (Reference number 666). Ninety-six well ELISA plates (Nunc, maxisorp) were 
coated with $3 \mu \mathrm{g} \mathrm{mAb47} \mathrm{in} \mathrm{carbonate} \mathrm{coating} \mathrm{buffer} \mathrm{per}$ well, overnight. After $3 \times 5$ min PBS $0.1 \%$ Tween (PBST) washes, wells were blocked with $2 \%$ BSA in PBST for $1 \mathrm{~h}$ and incubated overnight with CHO-TPO cell extracts in $2 \mathrm{mg} / \mathrm{ml}$ BSA in PBS. Wells were again washed and incubated overnight with either Fabs (diluted 1/1,000) or Graves' patient sera, individually diluted to $1 / 500$ in $2 \mathrm{mg} / \mathrm{ml}$ BSA in PBST. To study the effect of MMI, wells were incubated with MMI (500 nM-500 mM) for $30 \mathrm{~min}$ at $37^{\circ} \mathrm{C}$. Fab or serum Ig binding was detected with antihuman $\operatorname{IgG}$, Fab-specific Alk-Phos Conjugate raised in goat (Sigma).
Absorbance $\left(\mathrm{A}_{405}\right)$ was measured after $1 \mathrm{~h}$ incubation pNPP ELISA substrate (Sigma), using a 96-well-plate spectrophotometer (Ascent Multiskan).

Immunocytochemistry

All microscopy was performed using an Axioplan 2 widefield microscope with Zeiss Axiovision software. Cells were seeded after trypsin-passage onto $\left(1.8 \mathrm{~cm}^{2}\right)$ chamberslides (Nunc) and grown to near confluence (approximately 23 days). Media was changed to include MMI in complete

(a)

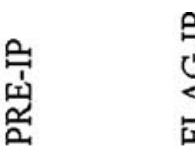

景
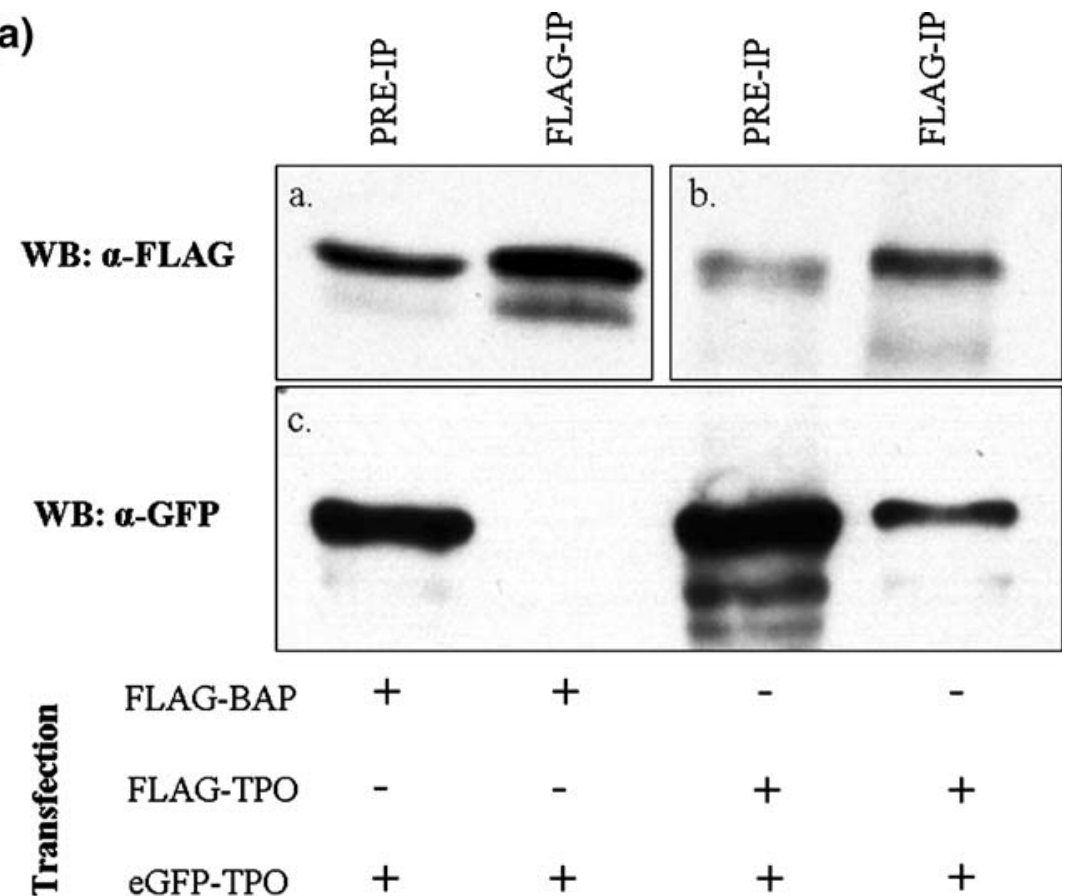
FLAG-BAP

$+$

FLAG-TPO

eGFP-TPO
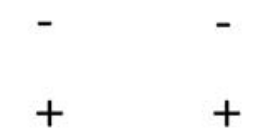

$+$

$+$

(b)
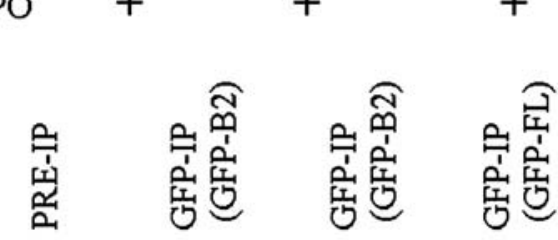

$+$

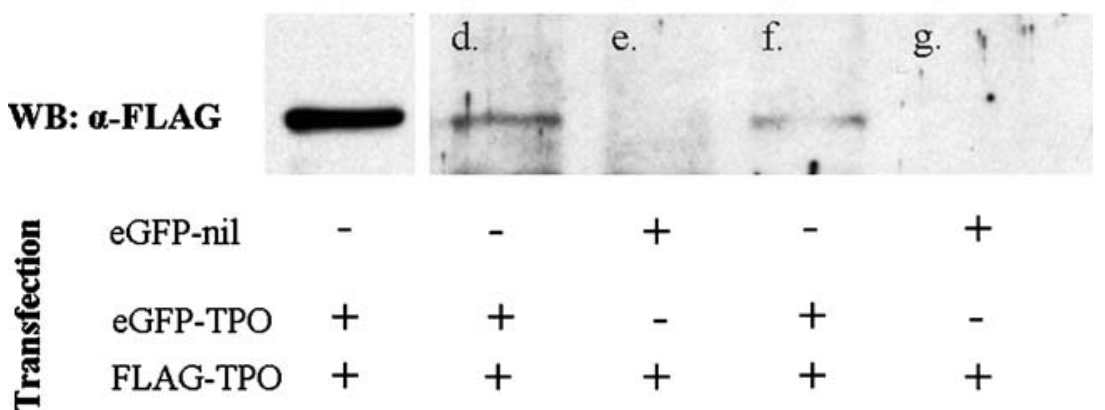

Fig. 2 Differentially tagged recombinant human thyroid peroxidase (rhTPO) forms dimeric complexes in Chinese hamster ovary (CHO) cells. CHO cells were co-transfected with FLAG- and enhanced green fluorescent protein (eGFP)-TPO, or their respective control constructs FLAG-BAP or eGFP-nil. Cell extracts were immunoprecipitated via their fusion-tags using either anti-FLAG M2, anti-GFP B2, or antiGFP FL antibody bound to protein-G sepharose. a FLAG-BAP (a.) and FLAG-TPO $(b$.) were immunoprecipitated using the anti-FLAG sepharose. Anti-GFP detected co-immunoprecipitating bands in the FLAG-TPO transfected cell extract but not following FLAG-BAP transfection (c.). b Both GFP antibodies co-immunoprecipitated FLAG-TPO along with eGFP-TPO $(d$. and $f$.) but not in cell extracts transfected with the eGFP-nil control construct (e. and $g$.) 
media as appropriate and cultured for a further $48 \mathrm{~h}$. Culture medium was then removed and cells immediately fixed with fresh $4 \%$ paraformaldehyde (PFA) on ice for $15 \mathrm{~min}$. Wells were washed three times with $\mathrm{PBS}+100 \mathrm{mM}$ glycine to remove residual PFA. After culture and fixation, appropriate wells were permeablised for 15 min with PBST (1\% Tween20). All wells were then blocked with $3 \%$ BSA in PBS for $1 \mathrm{~h}$ before incubation with primary antibody in $1 \%$ BSA $(1 / 50 \mathrm{mAb} 47)$ for a further hour. Wells were washed three times with PBS and incubated with anti-mouse $\operatorname{IgG}$ fluorescein isothiocyanate-conjugate (Sigma) for $1 \mathrm{~h}$. Wells were further washed three times, chambers removed, and vector shield with 4',6-diamidino-2-phenylindole (Vector Laboratories, UK) applied beneath a glass-coverslip.

\section{Statistical analysis}

Groups of measurements were compared using two-tailed $t$ tests, paired by the day of experiment.

\section{Results}

High-molecular weight TPO isoforms exist in CHO-TPO cells

Immunoblotting of extracts from the stably transfected CHO-TPO cell line with the monoclonal anti-TPO antibody mAb47 revealed a discrete $110 \mathrm{kDa}$ band and additional high-molecular weight (HMW) bands in the 220-240 kDa range. These HMW complexes were resistant to denaturation with conventional reducing agents (Fig. 1). The $110 \mathrm{kDa}$ isoform is consistent with the expected size of monomeric TPO. As MPO is known to function as a homodimer, we suspected that the HMW-TPO isoforms could also be homodimers.

High-molecular weight TPO is a homodimer

FLAG- and eGFP-tagged TPO were transiently co-expressed in $\mathrm{CHO}$ cells, and cell extracts were immunoprecipitated using antibody-conjugated protein-G sepharose beads. Cotransfections using FLAG-BAP and eGFP-nil, along with the tagged TPO constructs were used as controls. Cell extracts from cells co-transfected with FLAG- and eGFP-TPO revealed an eGFP reactive band following immunoprecipitation with anti-FLAG coated beads (Fig. 2). Similarly, immunoprecipitation of the same extracts with anti-GFP coated beads showed an immunoreactive band to anti-FLAG antibody. Co-transfected cells with either FLAG-BAP or eGFP-nil showed no non-specific binding (Fig. 2).

We also looked to confirm TPO dimerisation in an ELISA system, using cell extracts from FLAG-TPO and
eGFP-TPO co-transfected CHO cells. Plates were coated with anti-FLAG antibody, to capture FLAG-tagged TPO and probed with anti-GFP to detect TPO dimer. Extracts from FLAG-TPO and eGFP-nil co-transfected cells were used as a control. There was a clear detection of the GFP-tagged TPO when extracts of the FLAG-TPO and eGFP-tagged TPO co-transfected cells were studied (Fig. 3). Whereas, extracts from FLAG-TPO but eGFPnil co-transfected cells showed low signal when probed with anti-GFP $\mathrm{Ab}$. These results confirm that TPO homodimerises in this system and provides the likely explanation for the HMW-TPO isoforms seen on immunoblotting (Fig. 1).

Thionamide reduces high-molecular weight TPO expression in CHO-TPO cells

CHO-TPO cells were cultured in MMI or PTU, and cell extracts were subject to PAGE and immunoblotting. As before, the anti-TPO antibody $\mathrm{mAb} 47$ revealed bands of 110 and $220-240 \mathrm{kDa}$ from untreated CHO-TPO cells (Fig. 4). Cells grown in MMI for 10 days showed a reproducible and significant reduction in the HMW-TPO isoform(s). At an MMI concentration of $1 \mu \mathrm{M}$, there was a $58 \%$ reduction in HMW-TPO bands compared to untreated cells $(p=0.01)$, as normalised for GAPDH expression. Greater reductions in these HMW isoforms were found at higher MMI concentrations (58-82\% reduction, all $p<0.05$; Fig. 4). In contrast, there was no change in expression of the $110 \mathrm{kDa}$ TPO isoform during MMI treatment. The

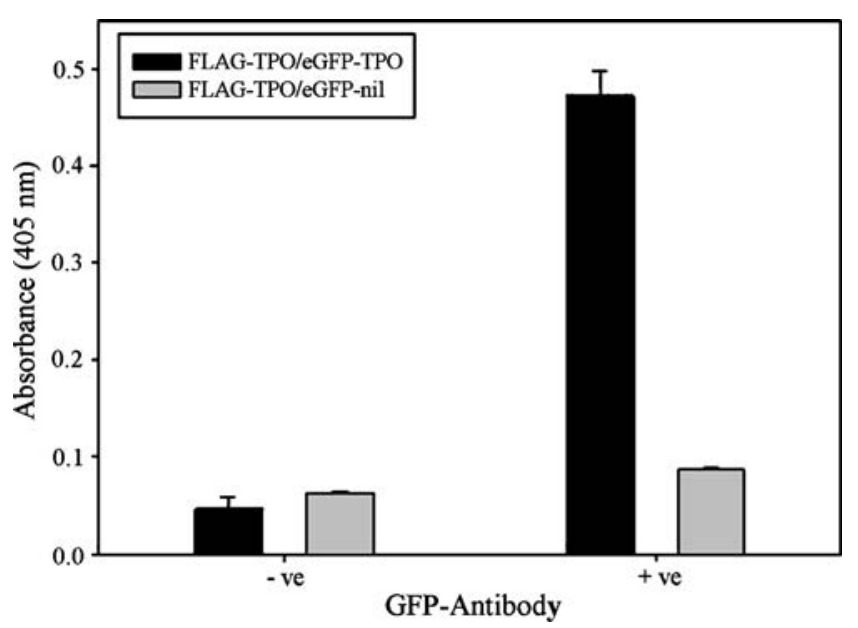

Fig. 3 Demonstration of dimeric thyroid peroxidase (TPO) by tagged TPO enzyme-linked-immunosorbent assay. FLAG-TPO from Chinese hamster ovary cells co-transfected with FLAG-TPO and enhanced green fluorescent protein (eGFP)-TPO, or eGFP-nil constructs was captured onto solid phase by murine anti-FLAG M2. A rabbit antiGFP (FL) antibody detected eGFP-TPO with an increase in the A405 of $>900 \%$ (mean of three replicates). This optical density was significantly greater than that observed in the absence of GFP antibody $(p<0.01)$ or than that of the GFP-nil control $(p<0.01)$ 
reduction in TPO-HMW isoforms could be observed after $24 \mathrm{~h}$ of culture in MMI $100 \mu \mathrm{M}$ and persisted for at least 10 days of continuous MMI exposure (data not shown).

As PTU is avidly protein bound, we cultured CHO-TPO cells in serum-free media with or without PTU to ensure maximal penetration into the cells. During $50 \mathrm{~h}$ exposure of CHO-TPO cells to PTU in serum-free conditions, we observed a similar, substantial reduction in HMW-TPO isoforms of between $34 \%$ and $74 \%$ across a PTU concentration gradient from 10 to $500 \mu \mathrm{M}(p<0.01$ at 100 and $500 \mu \mathrm{M}$; Fig. 4).

Effect of methimazole (MMI) on recombinant thyroid peroxidase epitope in vitro

We wished to see whether thionamide effects on TPO structure could alter its interaction with anti-TPO antibodies.
An ELISA system was developed to examine antibody binding to TPO by capturing rhTPO from CHO-TPO cell extracts with $\mathrm{mAb} 47$. Binding of sera from 36 TPO antibody positive AITD patients and from six TPO antibody negative healthy controls was examined in MMI concentrations from $500 \mathrm{nM}$ to $500 \mathrm{mM}$. There was no effect of increasing MMI concentration up to $50 \mathrm{mM}$ on binding of patient or control sera to captured rhTPO (Fig. 5). At the highest MMI concentration $(500 \mathrm{mM})$, there appeared to be a direct, possibly ionic, effect of the MMI on retention of antibodyTPO complex on the plate (washing and re-probing with mAb47 revealed no signal). Four Fabs, TR1.9, TR1.8, WR1.7, SP1.4, two each specific for the TPO immunodominant A or B regions, were tested in the ELISA system. Similar to the results with patient sera, we observed no substantial change in Fab binding to anchored TPO after treatment with MMI (500 $\mathrm{nM}$ to $50 \mathrm{mM}$; Fig. 5).

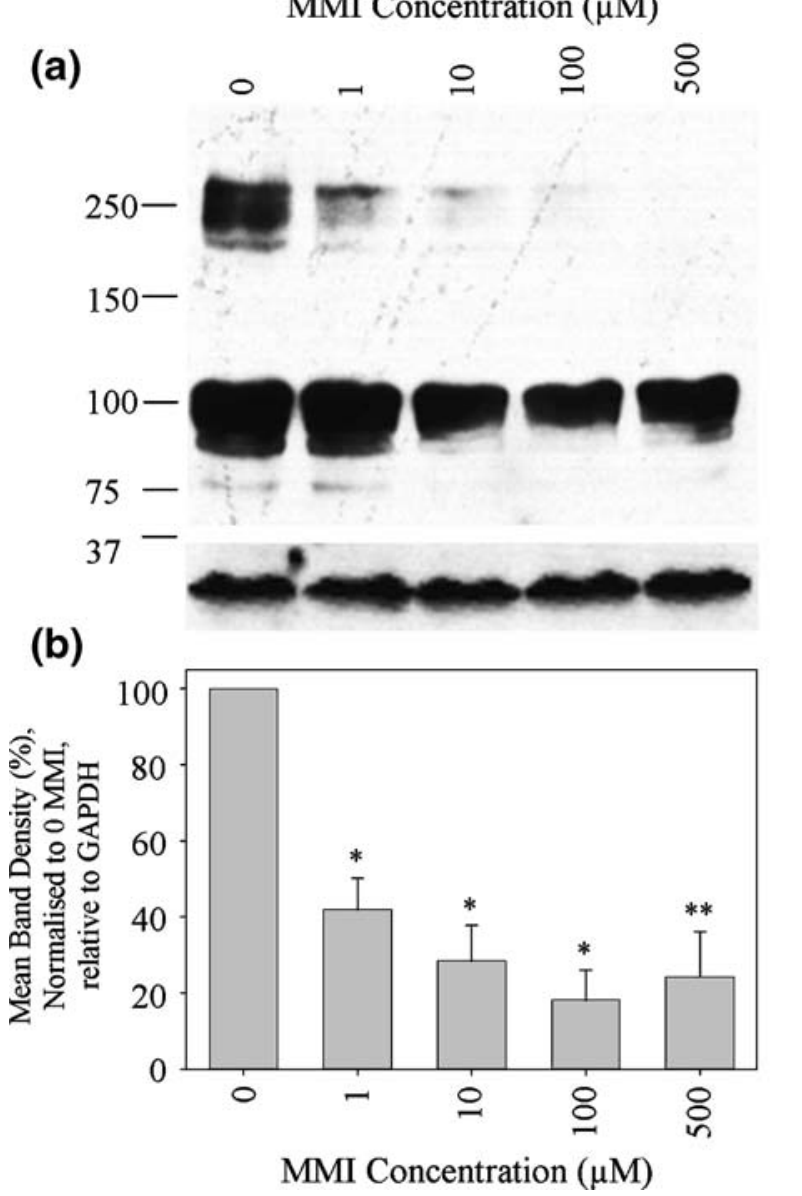

Fig. 4 High-molecular weight (HMW)-thyroid peroxidase (TPO) isoforms are reduced in Chinese hamster ovary (CHO)-TPO cultured with thionamide. Immunoblotting with mAb47 compared levels of recombinant human TPO (rhTPO) isoforms from CHO-TPO cells cultured in the presence of (a) methimazole (MMI) for 10 days or (c) propylthiouracil (PTU) for $50 \mathrm{~h}$ immediately prior to harvest. HMW $(>220 \mathrm{kDa})$ band densities were quantitated from triplicate experi-

\section{PTU Concentration $(\mu \mathrm{M})$}

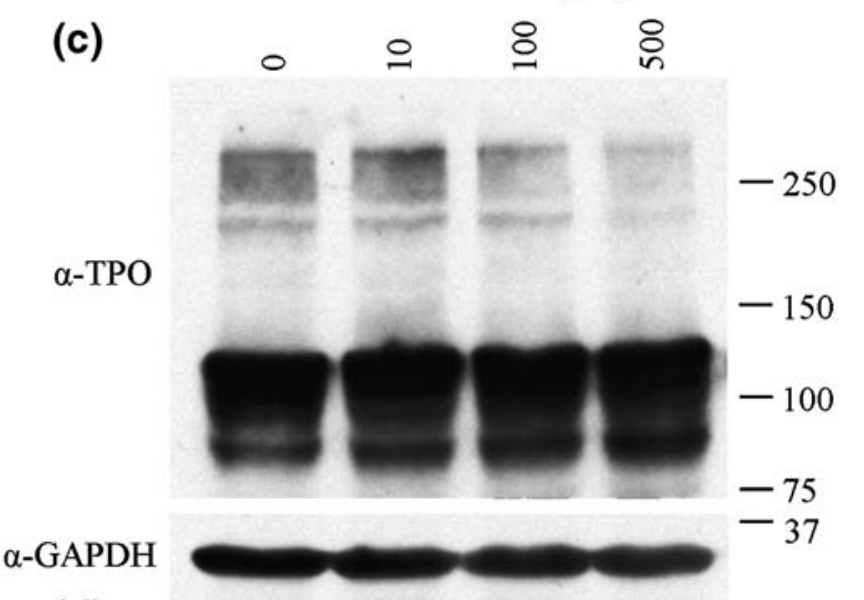

(d)

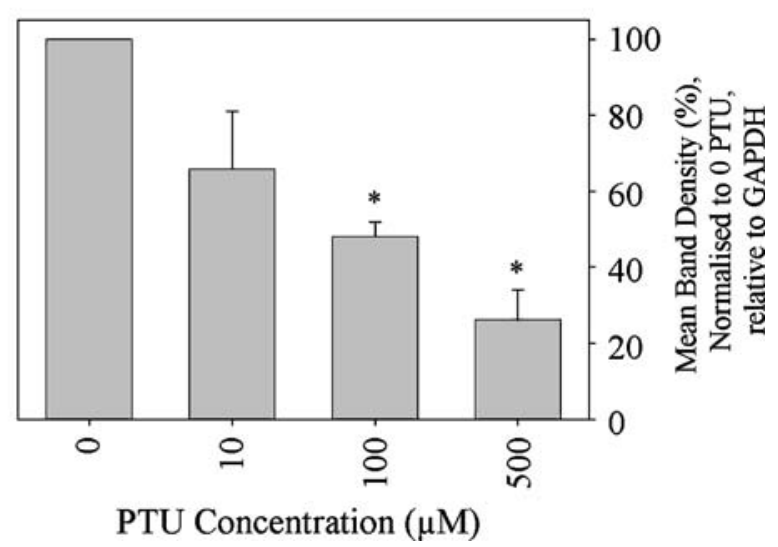

ments and normalised as a percentage of that observed in the absence of thionamide, relative to each sample's glyceraldehyde 3-phosphate dehydrogenase for both (b) MMI and (d) PTU. Highly significant reductions in HMW-TPO occurred at all MMI concentrations compared to the untreated condition $\left({ }^{*} p \leq 0.01, * * p<0.05\right)$. Likewise, lower levels of HMW isoforms were observed at all concentrations of PTU compared to the untreated condition 

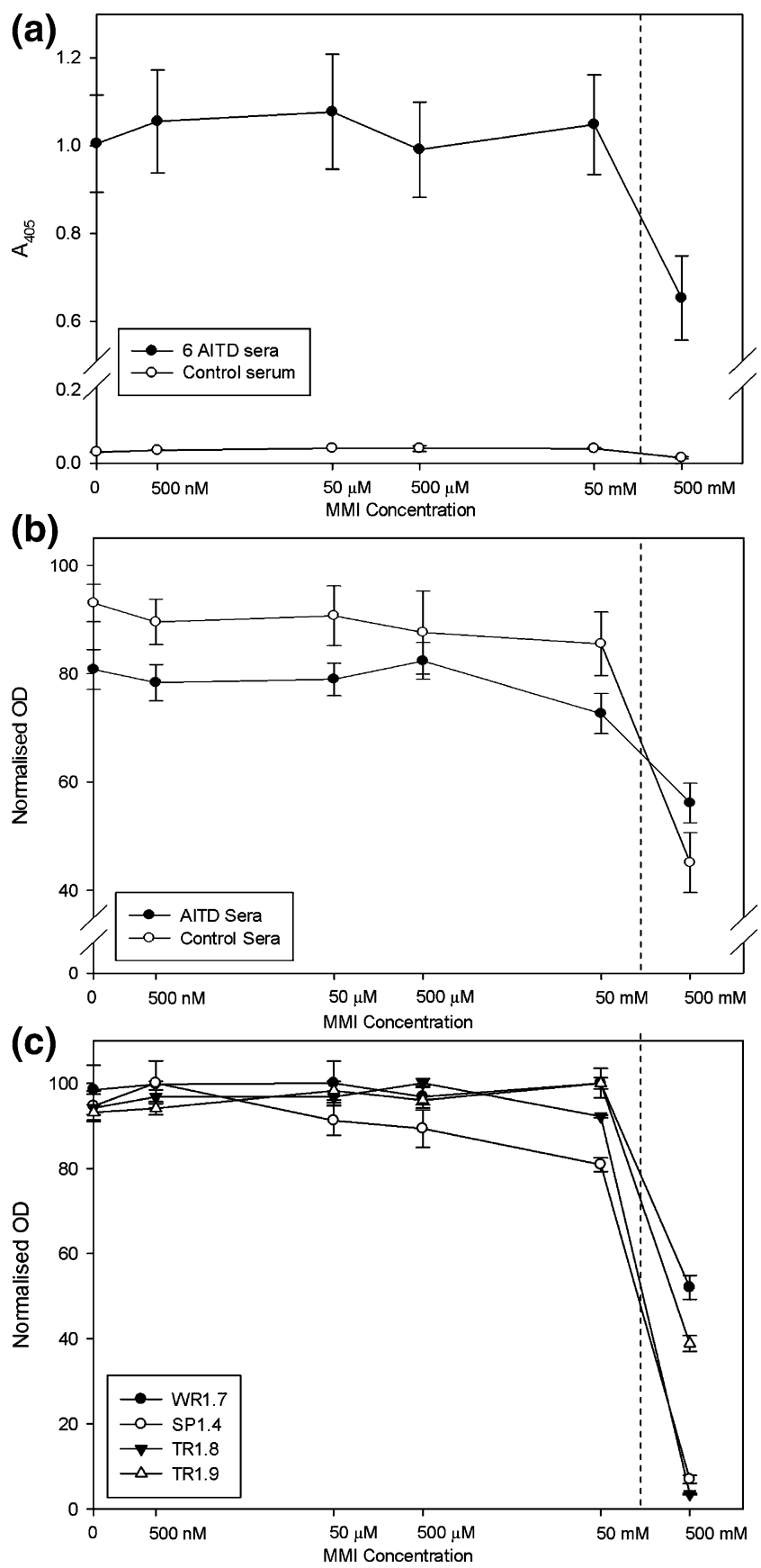

Effect of MMI on TPO localisation in CHO-TPO cells

Membrane localisation has been proposed as the site of enzymatically active mature TPO. We wondered whether thionamide could affect TPO localisation. CHO-TPO cells were grown in MMI at various pharmacologically relevant concentrations and studied by immunocytochemistry using mAb47. TPO expression, frequently in a punctate pattern, was seen both in a juxta-cell membrane location and in the cytosol in untreated CHO-TPO cells (Fig. 6). We observed no change in TPO distribution following exposure to MMI, either in unpermeabilised or permeabilised cells.
Fig. 5 Effect of methimazole (MMI) incubation on antibody binding to thyroid peroxidase (TPO). The effect of MMI on the binding of autoimmune thyroid disease (AITD) sera or immunodominant region (IDR)-specific Fabs to recombinant human TPO (rhTPO) was investigated by sandwich enzyme-linked-immunosorbent serologic assay incorporating a 30-min MMI incubation (500 nM-500 mM) following application of the sera or Fab. Substrate absorbance (measured as A405) was normalised as a percentage of the maximum observed for each serum or Fab, after non-specific binding to untransfected Chinese hamster ovary extracts was subtracted. a Representative absorbance data, from a single experiment, with six AITD patient sera and a single control. b Normalised data for 36 AITD patient sera and six normal control sera were included. No significant decrease (two-tailed $t$ test) in binding of Graves' or control sera at MMI concentrations up to $50 \mathrm{mM}$ was observed. c Four Fabs, specific for the TPO IDR were studied in triplicate. Following incubation with MMI at concentrations of $500 \mathrm{nM}-50 \mathrm{mM}$, there were no alterations in Fab binding to rhTPO. Substrate absorbance was dramatically reduced $(p \leq 0.01)$ at $500 \mathrm{mM}$ MMI in all experiments (we believe a non-specific effect, possibly related to high ionic content; see "Results"). Data presented as means with bars representing SEM

\section{Discussion}

We set out to explore the hypothesis that thionamides may influence the structure of TPO or its subcellular localisation. On Western analysis of stably transfected CHOTPO cells, we observed 220-240 kDa HMW-TPO bands, as well as the expected $110 \mathrm{kDa}$ monomeric band (Fig. 1). Although it is established that MPO, in its mature form, exists as a homodimer $[20,21]$ and it was suspected that TPO would similarly exist in a homodimeric state [22], our co-immunoprecipitation and ELISA binding studies with tagged recombinant TPOs (Figs. 2 and 3) confirm that TPO does indeed form dimeric complexes, at least in this system. Furthermore, we made the novel observation that these dimeric TPO isoforms are dramatically reduced by thionamide treatment, including both MMI and PTU, suggesting that these compounds interfere with the normal structural configuration of TPO. In vivo, thionamides accumulate in the human thyroid, with MMI reaching concentrations of $15 \mu \mathrm{M}$ during therapy for Graves' disease [23]. The response to MMI that we observed at in vitro concentrations as low as $1 \mu \mathrm{M}$ mean that this effect on TPO dimerisation is certainly plausible in vivo at the pharmacological concentrations that are used in Graves' disease. Although data on PTU concentrations in human thyroid is scanty, based on the equivalent potency of PTU to MMI, the higher concentrations of PTU at which we observed a similar reduction in HMW-TPO bands are also consistent with a relevant pharmacological effect in vivo. Nevertheless, our data on the PTU effect are less robust, as prolonged culture in serum-free conditions was not possible, and the intracellular concentration of PTU is likely to have been substantially influenced by protein binding. 

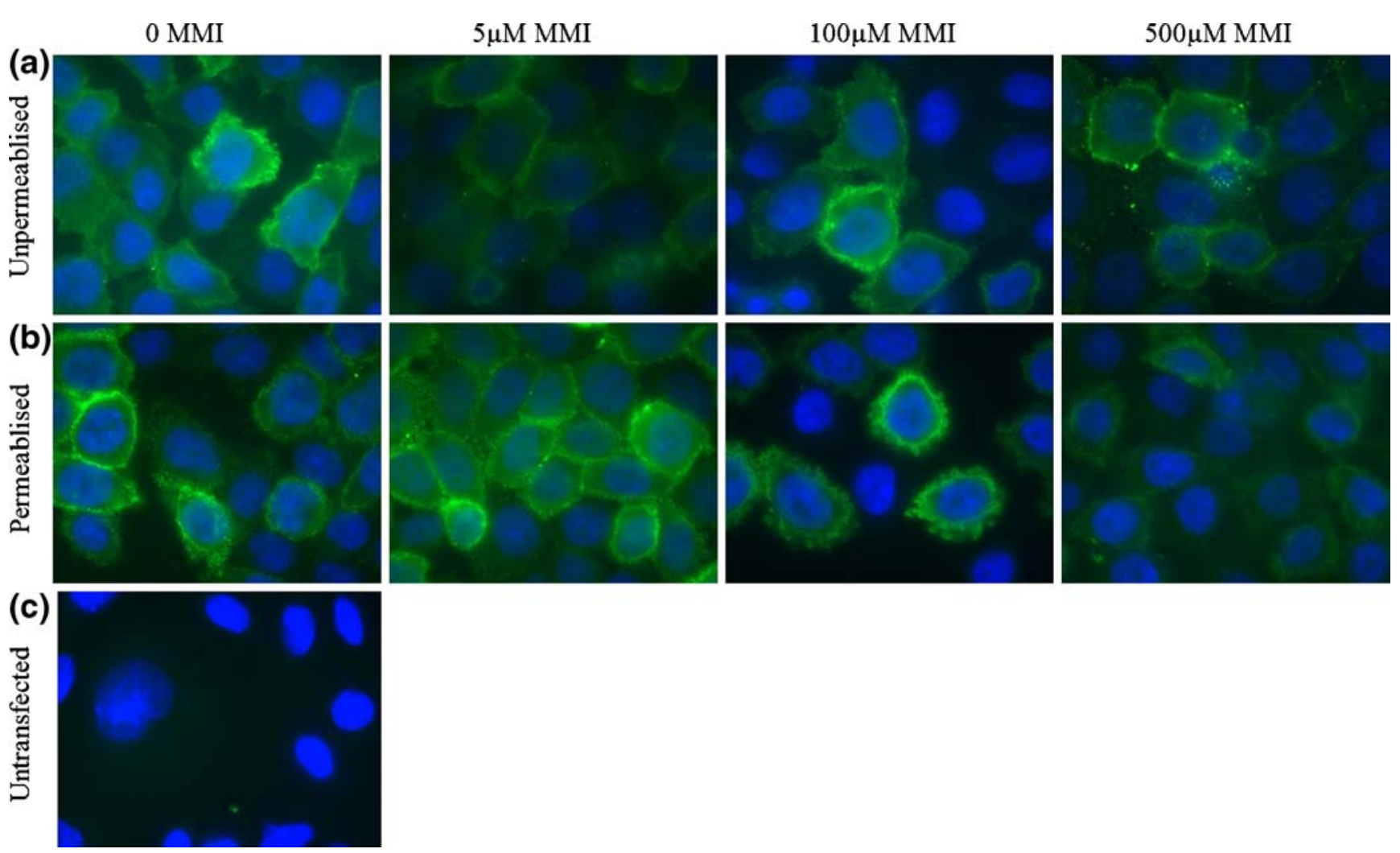

Fig. 6 Effect of methimazole (MMI) on localisation of thyroid peroxidase (TPO) in stably transfected Chinese hamster ovary (CHO) cells. CHO-TPO cells were seeded on coverslips and grown until near confluence, when in the media was changed to include/ exclude MMI $(5,100$, and $500 \mu \mathrm{M})$. After $24 \mathrm{~h}$, cells were fixed and left unpermeablised (a) or permeablised (b) with $0.1 \%$ Triton X-100 for $30 \mathrm{~min}$. TPO was detected using mab47 and visualised with anti-

In this study, we provide evidence that TPO exists in a homodimeric state and demonstrate that this dimerisation is sensitive to thionamide treatment. Therefore, we wondered if these observations could have relevance for thyroid physiology or the treatment of thyroid diseases. Firstly, it is possible that part of the inhibitory action of thionamides on TPO enzymatic activity in vivo is mediated by this druginduced change in structure. While this is certainly feasible, it is already known that the hemi-MPO components of disassociated MPO dimer retain their activity and that lactoperoxidase (LPO) exists and functions exclusively in a monomeric state [20, 24]. A second interesting hypothesis is that a change in the structure of the TPO conformational epitope could be responsible for the immunomodulatory effects of thionamide compounds in Graves' disease. This is an attractive hypothesis for several reasons. Firstly, TPO antibodies are practically universal in AITDs, are known to recognise distinct and conformational epitopes [3-6], and are able to mediate direct thyrocyte damage by fixing complement [25], with a plausible role in perpetuation of the immune attack in Graves' disease. Furthermore, thionamide compounds have established immunomodula- mouse IgG fluorescein isothiocyanate (green) conjugate at $\times 100$ magnification. Vectorshield mounting agent contained 4',6-diamidino2-phenylindole to counterstain DNA (blue). Untransfected CHO cells were included as a control (c). TPO showed juxta-cell membrane and cytosolic localization, in a punctate pattern. There was no major change in TPO localisation at any MMI concentration tested

tory effects in rodent autoimmune thyroiditis. Amelioration of thyroid infiltration and reduction of thyroid antibody production have been demonstrated in several models, including that induced by thyroglobulin in the mouse, by neonatal thymectomy in the Buffalo rat, and in spontaneous lymphocytic thyroiditis in the $\mathrm{BB} / \mathrm{W}$ rat [26-29]. These observations that thionamide compounds have immunomodulatory properties in autoimmune thyroiditides other than Graves' disease suggest that their immunomodulatory effect may be independent of hyperthyroidism and thus independent of the immune response to the TSHR. Lastly, because of the well-established interaction of thionamides with TPO (responsible for the antithyroid action), the principle of "Occam's razor" favours that the immunomodulatory action could be an additional consequence of the same TPO-thionamide interaction. Nevertheless, we cannot exclude that we are simply observing a non-specific reducing effect of thionamides on TPO dimeric isoforms and that similar chronic effects on other multimeric proteins might be responsible for immunomodulation through a mechanism unrelated to TPO. However, this does not seem a likely explanation given the low micromolar concen- 
trations of MMI with which we are able to demonstrate an influence on TPO structure.

Although it is clear that stimulating anti-TSHR antibodies are necessarily present in hyperthyroid Graves' disease, it is possible that they are neither sufficient nor the single aetiological cause of the condition. For instance, neonatal hyperthyroidism caused solely by transplacental passage of TSHR stimulating antibodies is a self-limiting disease without perpetuation of hyperthyroidism. In contrast to anti-TSHR antibodies, which typically circulate at nanograms per millilitre quantities in Graves' disease, antiTPO antibodies are found at 1,000-fold higher concentration [1]. In addition, anti-TPO antibodies have the capacity to fix complement and to mediate thyrocyte damage through antibody-dependent cell-mediated cytotoxicity $[25,30,31]$. Tantalisingly, there is already evidence that sera from AITD patients may preferentially recognise HMW-TPO isoforms [32, 33]. Thus, our finding that thionamides reduce TPO homodimerisation has the potential to be mechanistically significant. This idea that an autoimmune disease could be abrogated by a drug-induced structural change to an autoantigen has existing mechanistic parallels. It is already well accepted that an immune response can be generated against neoantigen formed by the interaction of a drug with a native protein, for example, in penicillin-induced autoimmune haemolytic anaemia [34]. The proposed mechanism is simply the complementary situation, in that the thionamide-TPO interaction could, in a proportion of individuals, disrupt the TPO conformational epitope to which the autoimmune response has already been directed. This 'removal' of an auto-epitope could lead to a reduced immune response and a subsequent amelioration of the disorder. It should also be noted that many autoantigens are enzymes or cell-surface receptors. These proteins already exist in at least two structural configurations, and thus their epitopes may naturally vary in structure depending upon the state of the receptor (liganded-unliganded) or enzyme (oxidised-reduced). Alternatively, the relative flexibility of their molecular structures may make them particularly susceptible to transformation into a novel conformational state following interaction with a drug.

Our ELISA experiments, using recombinant human TPO bound to the solid phase by mAb47 did not appear to show any effect of MMI on AITD serum or Fab binding to TPO in vitro. This may simply be that the drug effect requires dynamically folding nascent TPO proteins to disrupt the formation of the proper tertiary and quaternary structure. Thus, these effects may not be present in vitro. Alternatively, dimeric (HMW) TPO may be poorly bound to the ELISA plate, or the TPO epitope structure may have been stabilised by its binding to the anchoring mAb47 antibody. Similarly, we could find no evidence to support the straightforward possibility that in the presence of thiona- mide, TPO becomes mislocalised away from the cell surface. This finding is consistent with a previous study using MMI applied to native thyrocytes [35].

Our studies advance the field by demonstrating a molecular effect of thionamides on TPO macromolecular structure. We demonstrate, for the first time, that HMWTPO isoforms, which are likely to be dimeric TPO, are decreased in the presence of thionamide in vitro. This observation has possible relevance to a molecular mechanism for both the antithyroid thionamide action and for the immunomodulation of thyroid autoimmunity by these drugs. Additional work is needed both in vitro and in vivo to further illuminate these observations. The possibility of being able to design a novel antithyroid drug with greater immunomodulatory properties than the currently available thionamides, or of being able to predict relapse or remission of Graves' disease following drug treatment, based on a knowledge of antibody repertoire, would have substantial clinical implications.

Acknowledgements The authors thank Drs. Basil Rapoport and Sandy McLachlan for generously giving their advice and reagents in the conduct of these experiments. We also thank Dr. Monika Gora and Prof. J Paul Banga for kindly providing the CHO-TPO cell line, Prof. Jeremy Lakey for advice about structural biology, Tarana Singh-Dang for technical advice on co-immunoprecipitation, and Anna Mitchell for helpful comments on the manuscript. This work was funded by an MRC studentship to DOM.

Conflict of interest statement The authors declare that they have no conflict of interests.

Open Access This article is distributed under the terms of the Creative Commons Attribution Noncommercial License which permits any noncommercial use, distribution, and reproduction in any medium, provided the original author(s) and source are credited.

\section{References}

1. Beever K, Bradbury J, Phillips D, McLachlan SM, Pegg C, Goral A, Overbeck W, Feifel G, Smith BR (1989) Highly sensitive assays of autoantibodies to thyroglobulin and to thyroid peroxidase. Clin Chem 35:1949-1954

2. Libert F, Ruel J, Ludgate M, Swillens S, Alexander N, Vassart G, Dinsart C (1987) Thyroperoxidase, an auto-antigen with a mosaic structure made of nuclear and mitochondrial gene modules. EMBO J 6:4193-4196

3. Bresson D, Cerutti M, Devauchelle G, Pugniere M, Roquet F, Bes C, Bossard C, Chardes T, Peraldi-Roux S (2003) Localization of the discontinuous immunodominant region recognized by human anti-thyroperoxidase autoantibodies in autoimmune thyroid diseases. J Biol Chem 278:9560-9569

4. Guo J, McLachlan SM, Rapoport B (2002) Localization of the thyroid peroxidase autoantibody immunodominant region to a junctional region containing portions of the domains homologous to complement control protein and myeloperoxidase. J Biol Chem 277:40189-40195 
5. Gora M, Gardas A, Wiktorowicz W, Hobby P, Watson PF, Weetman AP, Sutton BJ, Banga JP (2004) Evaluation of conformational epitopes on thyroid peroxidase by antipeptide antibody binding and mutagenesis. Clin Exp Immunol 136:137-144

6. Bresson D, Rebuffat S, Peraldi-Roux S (2005) Localization of the immunodominant region on human thyroid peroxidase in autoimmune thyroid diseases: an update. J Autoimmune Dis 2:2

7. Abramowicz MJ, Targovnik HM, Varela V, Cochaux P, Krawiec L, Pisarev MA, Propato FV, Juvenal G, Chester HA, Vassart G (1992) Identification of a mutation in the coding sequence of the human thyroid peroxidase gene causing congenital goiter. J Clin Invest 90:1200-1204

8. Astwood E (1945) Chemotherapy of hyperthyroidism. Harvey Lect 40:195-235

9. Taurog A (1976) The mechanism of action of the thioureylene antithyroid drugs. Endocrinology 98:1031-1046

10. Solomon DH, Beck JC, Vanderlaan WP (1953) Prognosis of hyperthyroidism treated by antithyroid drugs. J Am Med Assoc 152:201-205

11. Weetman AP, McGregor AM, Hall R (1984) Evidence for an effect of antithyroid drugs on the natural history of Graves' disease. Clin Endocrinol (Oxf) 21:163-172

12. Reinwein D, Benker G, Lazarus JH, Alexander WD (1993) A prospective randomized trial of antithyroid drug dose in Graves' disease therapy. European Multicenter Study Group on Antithyroid Drug Treatment. J Clin Endocrinol Metab 76:1516-1521

13. McGregor AM, Petersen MM, McLachlan SM, Rooke P, Smith BR, Hall R (1980) Carbimazole and the autoimmune response in Graves' disease. N Engl J Med 303:302-307

14. Marcocci C, Chiovato L, Mariotti S, Pinchera A (1982) Changes of circulating thyroid autoantibody levels during and after the therapy with methimazole in patients with Graves' disease. J Endocrinol Investig 5:13-19

15. Weetman AP (1994) The immunomodulatory effects of antithyroid drugs. Thyroid 4:145-146

16. Volpe R (1994) Evidence that the immunosuppressive effects of antithyroid drugs are mediated through actions on the thyroid cell, modulating thyrocyte-immunocyte signaling: a review. Thyroid 4:217-223

17. Hosoya T, Kondo Y, Ui N (1962) Peroxidase activity in thyroid gland and partial purification of the enzyme. J Biochem 52:180-189

18. Magnusson RP, Chazenbalk GD, Gestautas J, Seto P, Filetti S, DeGroot LJ, Rapoport B (1987) Molecular cloning of the complementary deoxyribonucleic acid for human thyroid peroxidase. Mol Endocrinol 1:856-861

19. Nishikawa T, Costante G, Prummel MF, McLachlan SM, Rapoport B (1994) Recombinant thyroid peroxidase autoantibodies can be used for epitopic "fingerprinting" of thyroid peroxidase autoantibodies in the sera of individual patients. J Clin Endocrinol Metab 78:944-949

20. Andrews PC, Krinsky NI (1981) The reductive cleavage of myeloperoxidase in half, producing enzymically active hemimyeloperoxidase. J Biol Chem 256:4211-4218
21. Fiedler TJ, Davey CA, Fenna RE (2000) X-ray crystal structure and characterization of halide-binding sites of human myeloperoxidase at 1.8. A resolution. J Biol Chem 275:11964-11971

22. Hamada N, Portmann L, DeGroot LJ (1987) Characterization and isolation of thyroid microsomal antigen. J Clin Invest 79:819-825

23. Jansson R, Dahlberg PA, Johansson H, Lindstrom B (1983) Intrathyroidal concentrations of methimazole in patients with Graves' disease. J Clin Endocrinol Metab 57:129-132

24. Singh AK, Singh N, Sharma S, Singh SB, Kaur P, Bhushan A, Srinivasan A, Singh TP (2008) Crystal structure of lactoperoxidase at 2.4. A resolution. J Mol Biol 376:1060-1075

25. Khoury EL, Hammond L, Bottazzo GF, Doniach D (1981) Presence of the organ-specific 'microsomal' autoantigen on the surface of human thyroid cells in culture: its involvement in complement-mediated cytotoxicity. Clin Exp Immunol 45:316328

26. Davies TF, Weiss I, Gerber MA (1984) Influence of methimazole on murine thyroiditis. Evidence for immunosuppression in vivo. $\mathrm{J}$ Clin Invest 73:397-404

27. Cohen SB, Weetman AP (1988) Antithyroid drugs ameliorate thymectomy-induced experimental autoimmune thyroiditis. Autoimmunity 1:51-58

28. Rennie DP, McGregor AM, Keast D, Weetman AP, Foord SM, Dieguez C, Williams ED, Hall R (1983) The influence of methimazole on thyroglobulin-induced autoimmune thyroiditis in the rat. Endocrinology 112:326-330

29. Allen EM, Rajatanavin R, Nogimori T, Cushing G, Ingbar SH, Braverman LE (1986) The effect of methimazole on the development of spontaneous lymphocytic thyroiditis in the diabetes-prone BB/W rat. Am J Med Sci 292:267-271

30. Bogner U, Schleusener H, Wall JR (1984) Antibody-dependent cell mediated cytotoxicity against human thyroid cells in Hashimoto's thyroiditis but not Graves' disease. J Clin Endocrinol Metab 59:734-738

31. Guo J, Jaume JC, Rapoport B, McLachlan SM (1997) Recombinant thyroid peroxidase-specific Fab converted to immunoglobulin $\mathrm{G}$ (IgG) molecules: evidence for thyroid cell damage by IgG1, but not IgG4, autoantibodies. J Clin Endocrinol Metab 82:925931

32. Kaufman KD, Rapoport B, Seto P, Chazenbalk GD, Magnusson RP (1989) Generation of recombinant, enzymatically active human thyroid peroxidase and its recognition by antibodies in the sera of patients with Hashimoto's thyroiditis. J Clin Invest 84:394-403

33. Baker JR, Arscott P, Johnson J (1994) An analysis of the structure and antigenicity of different forms of human thyroid peroxidase. Thyroid 4:173-178

34. Levine BB (1966) Immunologic mechanisms of penicillin allergy. A haptenic model system for the study of allergic diseases of man. N Engl J Med 275:1115-1125

35. Aguayo J, Michaud P, Iitaka M, Degroot LJ, Row VV, Volpe R (1989) Lack of effect of methimazole on thyrocyte cell-surface antigen expression. Autoimmunity 2:133-143 